\title{
A Community Perspective on Bowel Management and Quality of Life after Spinal Cord Injury: The Influence of Autonomic Dysreflexia
}

\author{
Jessica A. Inskip,, ${ }^{1, *}$ Vera-Ellen M. Lucci,, ${ }^{1, *}$ Maureen S. McGrath, \\ Rhonda Willms, ${ }^{2-4}$ and Victoria E. Claydon ${ }^{1,2}$
}

\begin{abstract}
Autonomic dysfunction is common in individuals with spinal cord injury (SCI) and leads to numerous abnormalities, including profound cardiovascular and bowel dysfunction. In those with high-level lesions, bowel management is a common trigger for autonomic dysreflexia (AD; hypertension provoked by sensory stimuli below the injury level). Improving bowel care is integral for enhancing quality of life $(\mathrm{QoL})$. We aimed to describe the relationships between bowel care, AD, and QoL in individuals with SCI. We performed an online community survey of individuals with SCI. Those with injury at or above T7 were considered at risk for AD. Responses were received from 287 individuals with SCI (injury levels C1-sacral and average duration of injury $17.1 \pm 12.9$ [standard deviation] years). Survey completion rate was $73 \%(n=210)$. Bowel management was a problem for $78 \%$ : it interfered with personal relationships $(60 \%)$ and prevented staying $(62 \%)$ and working $(41 \%)$ away from home. The normal bowel care duration was $>60 \mathrm{~min}$ in $24 \%$ and most used digital rectal stimulation (59\%); 33\% reported bowel incontinence at least monthly. Of those at risk for AD $(n=163), 74 \%$ had AD symptoms during bowel care; $32 \%$ described palpitations. AD interfered with activities of daily living in 51\%. Longer durations of bowel care $(p<0.001)$ and more severe $\mathrm{AD}(p=0.04)$ were associated with lower QoL. Bowel management is a key concern for individuals with SCI and is commonly associated with symptoms of AD. Further studies should explore ways to manage bowel dysfunction, increase self-efficacy, and ameliorate the impact of AD to improve QoL.
\end{abstract}

Keywords: autonomic dysreflexia; bowel management; cardiac arrhythmia; orthostatic hypotension; quality of life; spinal cord injury

\section{Introduction}

A PPROXIMATELY 2.5 MILLION PEOPLE worldwide are living with the devastating consequences of spinal cord injury (SCI). ${ }^{1,2}$ In addition to well-known motor and sensory consequences of SCI, descending spinal autonomic pathways can also be affected, leading to profound autonomic dysfunction. ${ }^{3-5}$ Although the autonomic consequences of SCI are widespread, key areas of concern identified by individuals living with SCI relate to issues regarding continence and cardiovascular dysfunction. ${ }^{6}$ These devastating effects are major problems for individuals with SCI: they represent frustrating and limiting secondary complications that are too often forgotten by the clinical and research communities. Indeed, improving bowel func- tion has been identified by individuals with SCI, both those with tetraplegia and those with paraplegia, as a key target in order to enhance quality of life (QoL) ${ }^{6-8}$ Notably, issues surrounding bowel management are complicated by cardiovascular dysfunction that is triggered by the bowel care itself.

Bowel management concerns post-SCI are multifactorial, but most commonly relate to neurogenic bowel, a colonic dysfunction resulting from lack of central nervous system control. ${ }^{7,9-11}$ Accordingly, individuals with SCI often experience impairments in QoL related to fecal incontinence and fecal urgency, constipation, hemorrhoids, and abdominal distention. ${ }^{7,9-11}$ Changes in bowel motility, sphincter control, and impaired hand function and mobility combine to make bowel management a key cause of

\footnotetext{
${ }^{1}$ Department of Biomedical Physiology and Kinesiology, Simon Fraser University, Burnaby, British Columbia, Canada.

${ }^{2}$ International Collaboration On Repair Discoveries (ICORD), University of British Columbia, Vancouver, British Columbia, Canada.

${ }^{3}$ GF Strong Rehabilitation Center, Spinal Cord Injury Program, Vancouver Coastal Health, Vancouver, British Columbia, Canada.

${ }^{4}$ Division of Physical Medicine and Rehabilitation, Faculty of Medicine, UBC, Vancouver, British Columbia, Canada.

*These authors contributed equally to this work.
}

(C) Jessica A. Inskip et al. 2018; Published by Mary Ann Liebert, Inc. This Open Access article is distributed under the terms of the Creative Commons Attribution Noncommercial License (http://creativecommons.org/licenses/by-nc/4.0/) which permits any noncommercial use, distribution, and reproduction in any medium, provided the original author(s) and the source are credited. 
morbidity post-SCI. ${ }^{7,9-11}$ Successful bowel management requires a multi-factorial approach, with important roles for dietary approaches, fiber supplementation, adequate fluid consumption, routine bowel practice, and often the use of suppositories, prokinetics, osmotic agents, or digital rectal stimulation in combination with abdominal massage. ${ }^{7,9-11}$ Appropriate timing, use of assistive devices, positioning, and personal assistance are also components of successful bowel management. ${ }^{10,12}$

One factor that impinges on bowel management for individuals with SCI is the impact of injury to descending spinal autonomic (sympathetic) pathways on cardiovascular function - the severity of autonomic injury. ${ }^{13-17}$ Disrupted cardiovascular function post-SCI leads to numerous cardiovascular abnormalities, and cardiovascular disease is a leading cause of morbidity and mortality post-SCI. ${ }^{18-20}$ Of particular concern is the phenomenon of autonomic dysreflexia (AD), in which sudden and extreme hypertension is elicited by afferent stimuli originating from below the lesion level. These episodes can be life-threatening and may result in vascular dysfunction, ${ }^{21}$ seizures, ${ }^{22-24}$ cardiac arrest, ${ }^{25}$ cerebral vascular accidents, ${ }^{22,26-29}$ and death. ${ }^{22,23,26,28} \mathrm{AD}$ is described in up to $90 \%$ of individuals with tetraplegia or high paraplegia ${ }^{28,30}$ and only occurs with lesions above the level of sympathetic outflow to the splanchnic vascular bed (the fifth thoracic level), the most important vascular resistance and capacitance region and a key site for blood pressure regulation. ${ }^{31}$

$\mathrm{AD}$ can occur in response to any afferent stimulus below the lesion level, ${ }^{31}$ but the most potent triggers are visceral stimuli, ${ }^{30}$ such as during bowel care. ${ }^{32}$ The blood pressure rise is secondary to vasoconstriction in resistance and capacitance vessels below the lesion, attributed to reflex sympathetic activity triggered by afferent input to the isolated spinal cord that proceeds unchecked by regulation from injured descending spinal autonomic pathways. ${ }^{33}$ The cardiac vagus nerve does not pass through the spinal cord, so it is unaffected by SCI, producing a baroreflex-mediated increase in cardiac vagal stimulation ${ }^{15}$ coincident with the high cardiac sympathetic outflow. Many individuals with AD also experience concurrent palpitations attributed to cardiac arrhythmia triggered by the combined high cardiac vagal and sympathetic outflow during AD. ${ }^{14,17,34,35}$

Little is known about the associations between cardiovascular dysfunction and bowel care post-SCI. A recent survey found that $45 \%$ of those with injuries above the seventh thoracic level experienced $\mathrm{AD}$ during their usual bowel care, which was identified as experiencing headache or sweating. ${ }^{8}$ However, this is likely a gross underestimation of the scope of the problem-not all episodes of $\mathrm{AD}$ present with these symptoms, and $\mathrm{AD}$ can be asymptomatic. ${ }^{36}$ Incidence of cardiac arrhythmia associated with AD during bowel care is unknown. In contrast to the hypertension experienced during $\mathrm{AD}$, many individuals with high-level SCI (at or above T5) have profound hypotension between episodes, again attributed to the disruption of autonomic regulation of key sites for blood pressure control in the splanchnic vascular bed. These hypotensive episodes are exacerbated when in the upright posture (orthostatic hypotension; $\mathrm{OH}$ ) and lead to disabling symptoms of dizziness and fatigue.

While it is recognized that bowel care is a concern for individuals with $\mathrm{SCI},{ }^{6-8}$ the relationships between bowel care, cardiovascular dysfunction, and domains of QoL are poorly understood. This makes it challenging to target components of bowel care that might be modified to improve QoL for those affected. We sought to conduct a survey of the experts in this area-individuals living with SCI - to identify common bowel practices used by individuals with SCI, describe the presence of cardiovascular symptoms during bowel care, and elucidate the impact of bowel management and associated cardiovascular concerns on QoL.

\section{Methods}

\section{Participants}

This study was approved by the Department of Research Ethics at Simon Fraser University (Burnaby, British Columbia, Canada) and conforms to the principles outlined in the Declaration of Helsinki. ${ }^{37}$ Participants were recruited using a multi-method approach. Postcards advertising the study were distributed at local rehabilitation and community centers in Greater Vancouver, Canada. Online advertising for the study was posted on our institution website (www.icord.org) as well as discussion forums and online community groups specific to SCI, including: Inspired Spinal Cord Injury Support Community (inspiredsciforum.com); Care Cure Community (sci.rutgers.edu); Spinal Injury Network (spinal-injury.net); Apparelyzed (apparelyzed.com); and Spinal Cord Injury British Columbia (facebook.com/SpinalCordInjuryBC). Information about the survey was also e-mailed to 250 randomly selected Englishspeaking individuals who had indicated they wished to be contacted about ongoing SCI research and were members of a mailing list owned by the Rick Hansen Institute (rickhanseninstitute.org).

The convenience sample of participants self-administered either a paper-based or online survey (fluidsurveys.com/s/bowelcare-SCI; Supplementary Appendix A) (see online supplementary material at http://www.liebertpub.com). To begin the survey, participants received some basic information about the study (Supplementary Appendix A) (see online supplementary material at http://www. liebertpub.com) gave consent (by clicking a link in the online version or by submitting the responses in a pre-addressed envelope for the paper-based version) and acknowledged that they had sustained a SCI and were over 18 years old. Paper copies were administered and collected from a local rehabilitation facility.

\section{Questionnaire design}

The Bowel care and cardiovascular function after spinal cord injury questionnaire (Supplementary Appendix A) (see online supplementary material at http://www.liebertpub.com) was designed using the International Bowel Function Basic and Extended Data Sets, developed by a working group including members from the American Spinal Injury Association and the International Spinal Cord Society. ${ }^{38,39}$ Additional questions were incorporated from another recent community-based bowel survey. ${ }^{8}$ Questions about cardiovascular signs and symptoms were included based on previous work in individuals with SCI. ${ }^{40}$ Basic questions about nutrition, hydration, medication use, and participant demographics were also included. Due to the personal nature of some questions, and the potential for participants with impaired hand function to need assistance in completing the survey, it was not required for individuals to complete all items.

\section{Measurements}

When considering the measurements derived, we denote relevant survey question numbers as noted in Supplementary Appendix A (see online supplementary material at http://www.liebertpub .com) using the abbreviation Q[number].

Neurological level and completeness of spinal cord injury. Respondents self-reported their neurological level of injury (Q1). Motor and sensory completeness of injury was determined by respondent's answers to the following questions: 1) Can you feel touch in your anal area? (Q2); 2) Can you voluntarily tighten your anal sphincter? (Q3); 3) Can you feel light touch below your lesion level? (Q4); 4) Can you feel the difference between sharp and dull below your lesion level? (Q5); and 5) Can you lift your legs against gravity? (Q6) If the answer to all of these questions was "no," then an individual was considered to have a motor and sensory complete injury. Use of mobility aids was considered (Q7-8). 
Bowel management. Basic bowel management information (method used, medication use, positioning during bowel care, level of assistance required, typical duration of routine, frequency and timing of bowel care, experiences of fecal incontinence, and satisfaction with their bowel care) was collected using questions from the International Bowel Function Basic and Extended Data Sets ${ }^{39,41}$ and previous surveys (Q9-29). ${ }^{8}$ Participants were also asked how bowel management fits into their life (Q31). Participants were asked questions about their fluid intake and use of diuretics (Q50-55).

Quality of life. As a test of internal validity, QoL with respect to bowel management was evaluated in two different ways. Participants were asked to rank qualitatively whether their bowel management has "No impact," "Little impact," "Some impact," or "Major impact" on their QoL (Q30). Participants were asked to rank the impact of managing their bowel on QoL compared to other aspects of their injury (sexual function, pain, bladder management, spasticity, using a wheelchair for mobility, and skin concerns) on a scale from zero (0) to ten (10), with ten being the worst effect and zero being the least effect (Q32). ${ }^{8}$ This continuous measure was taken to be their overall QoL with respect to their bowel management. A larger score was indicative of a poorer QoL, whereas a lower score indicates a greater QoL.

Cardiovascular symptoms. Participants were asked about whether they had experienced AD and $\mathrm{OH}$, and how often (Q3637; Q42-45). Five-point ordered Likert scales were used to assess symptoms of $\mathrm{AD}$ during bowel care, and at other times of the day (Q35, Q38). Frequencies of AD symptoms were assessed using a range of "Never," "Rarely," "Monthly," "Weekly," and "Daily," whereas severity was assessed as "Not experienced," "Mild," "Moderate," "Severe," and "Very severe." When considering symptoms of $\mathrm{AD}$ during bowel care, in addition to considering the full item response, symptoms were also considered in a dichotomized form independent of severity as "experienced" (mild, moderate, severe, or very severe symptoms reported) and "not experienced" (no symptoms experienced). We also asked about symptoms of $\mathrm{AD}$ in response to 12 other common $\mathrm{AD}$ stimuli (Q39) and provided an opportunity for individuals to add a category "other" to identify additional stimuli. An overall symptom severity score of $\mathrm{AD}$ both overall (Q38) and during bowel care (Q35) was determined by assigning numerical values to the Likert scale ("Never" or "Not experienced" =0; "Rarely" or "Mild" = 1; "Monthly" or "Moderate" = 2; "Weekly" or "Severe" =3; and "Daily" or "Very severe" =4) and summing the responses to individual symptoms. "Other" symptoms were scored in a similar way where identified and incorporated in the overall symptom score, because we were particularly interested in the overall symptom burden of $\mathrm{AD}$. Use of "boosting" through deliberate initiation of $\mathrm{AD}$ was considered (Q41).

Sixteen common symptoms of $\mathrm{OH}$ were also assessed using the same frequency range and severity (Q46). These symptoms were also assigned a composite severity score in the same manner as the AD data described above. We also asked about symptoms of $\mathrm{OH}$ in response to 10 other common $\mathrm{OH}$ stimuli (Q47) and provided an opportunity for individuals to add a category "other" to identify additional stimuli. Participants were asked about their fainting history (Q42-45).

Participants were asked whether symptoms of $\mathrm{OH}$ or $\mathrm{AD}$ had ever interfered in their ability to participate in common activities (Q40, Q48; activities of daily living, work, exercise, sexual activity, rehabilitation, household chores, driving, and social activities). A category "other" was available for participants to identify additional activities.

Participants were asked if they experienced symptoms of cardiac arrhythmia (heart palpitations, irregular heartbeats, or a feeling of "fluttering in the chest" during bowel care) (Q33), and, if yes, how often this occurred (daily, weekly, monthly, rarely, or never; Q34).

Fatigue was score on a linear scale ( 0 , no fatigue; 10 severe fatigue; Q49). ${ }^{42}$

\section{Stratification of participants by injury level}

For analysis of cardiovascular symptoms, participants were stratified by their self-reported injury level into two subgroups: those with injury at or above the seventh thoracic segment (T7) and those with injuries below T7. High-level injuries can disrupt the sympathetic control of the heart and the vasculature in the splanchnic bed, increasing susceptibility to $\mathrm{OH}, \mathrm{AD}$, and cardiac arrhythmia. In an effort to account for uncertainty regarding precise injry details with self-reported data, and physiological variability in autonomic pathways, the cut-off for defining an individual at risk for cardiovascular abnormalities was set conservatively, at T7.

Data on frequency of bowel incontinence were considered separately for those with an upper motor neuron neurogenic bowel disorder (those with injury at or above L1) and those with a lower motor neuron neurogenic bowel disorder (those with injury below L1).

\section{Statistical analyses}

Data processing and statistical analyses were performed on JMP (Version 12.0.1; SAS Institute Inc., Cary, NC), R (Version 3.0.2; R Foundation for Statistical Computing, Vienna, Austria), and RStudio software (Version 0.98.507; RStudio, Boston, MA). Due to the sensitive nature of the questions, it was not required for individuals to complete the survey in its entirety, with skipping questions or "not applicable" as possible options; thus, not all respondents completed all question or subquestion items. Accordingly, responses are expressed as the percentage of responses received for each item, followed by the number of item responses expressed as a proportion of the total responses received for that question (percent [item response/question response]). Comparisons of proportions between subgroups were made using Fisher's exact test. Interval data were compared using Wilcoxon rank-sum tests, with Bonferonni correction for repeated tests. Chi-squared tests were used to compare the distribution of cardiovascular symptoms and triggers between SCI groups. Post-hoc comparisons were made using pair-wise Fisher's exact tests. To evaluate predictors of $\mathrm{OH}$, we considered the following factors: level of injury (coded as a linear scale with $\mathrm{C} 1=1, \mathrm{C} 2=2$, etc.); age; duration of injury; fatigue score (linear scale from zero [no fatigue] to ten [severe fatigue]); amount of AD experienced daily; and whether respondents intentionally used fluid restriction to aid in bladder management (coded on the linear scale as "No" =1; "Yes, but rarely" =2; "Yes, sometimes" =3; and "Yes, often" =4). Linear regression models were then performed to establish predictors of $\mathrm{OH}$. To evaluate potential predictors of the impact of bowel care on QoL, we considered the following factors: duration of injury; level of injury; number of bowel management approaches used; duration with current routine (number of years using the current bowel management approach); time to complete current routine (time in minutes to complete a single bowel care routine); frequency of bowel incontinence (coded on a linear scale as "Never" = 1; "Less than once per month" =2; "Once per month" = 3; "Not every week but at least once per month" $=4$; "Not every day but at least once per week" =5; "Once daily" =6; and "Two or more episodes per day" =7); severity of AD symptoms during routine; fatigue score; and whether respondents intentionally used fluid restriction to aid in bladder management. As an initial step, we performed simple unadjusted correlations (Spearman's $r$ ) between these possible variables of interest with QoL, and with each other as potential confounds. Multiple linear regression models were then performed to establish the predictors of $\mathrm{QoL}$ when potential confounds were considered. These regression models included the following factors: duration or injury; level of injury; number of bowel management approaches used; duration with current routine; time to complete current routine; frequency of bowel incontinence; severity of $\mathrm{AD}$ symptoms during routine; fatigue score; and whether 
respondents intentionally used fluid restriction as independent variables. Significant predictors were determined to be primary predictors of QoL. Secondary predictors of QoL were determined by linear regression models including all above independent variables run against primary predictors of QoL. Data were included in the regression analyses where all parameters were available for each individual - where questions relating to parameters of interest were skipped, data for that individual were not incorporated in that particular regression model. Akaike information criterion (AIC) variable importance analysis was performed to determine the relative importance of each approach to bowel management. A score $\geq 0.8$ was considered to indicate factors with a high variable importance. Statistical significance was assumed at a $p$ value of $<0.05$. Where appropriate, data are presented as mean \pm standard error (SE), unless otherwise stated.

\section{Results}

A total of 287 participants submitted survey responses over a 3-year time span. The majority (73\% [210 of 287]) of respondents completed all survey items. Respondents primarily originated from Canada (55\% [158 of 287]), with the remainder from the United States ( $8 \%$ [23 of 287]), Europe (6\% [18 of 287]), New Zealand ( $1 \%$ [2 of 287]), Columbia (1\% [2 of 287]), India (<1\% [1 of 287]), and China $(<1 \%$ [ 1 of 287]). The location of $29 \%$ [82 of 287] of respondents was unknown.

\section{Demographic and injury information}

Demographic and injury information can be seen in Table 1. There was a wide range of respondent ages (20-84 years), time postinjury ( 8 months -49 years), and injury levels (C1-sacral). Approximately two thirds of respondents (67\% [163 of 245]) had an injury at or above T7, and the majority (63\% [143 of 228]) of injuries were incomplete.

\section{General bowel management}

A summary of general bowel management practices can be found in Table 2. Most respondents completed their bowel care in the morning (53\% [113 of 215]), at least once per day (51\% [118

Table 1. Participant Demographic and Injury Information

Demographic and injury information

\begin{tabular}{lc}
\hline Sample size $(n)$ & 287 \\
Age (years) & $49.2 \pm 13.2$ \\
Time post-injury (years) & $17.1 \pm 12.9$ \\
Injury level (\%) & \\
$\quad$ Cervical & 45 \\
Thoracic & 45 \\
Lumbar & 9 \\
Sacral & 1 \\
Injury completeness (\%) & \\
Complete & 30 \\
Incomplete & 70 \\
Cardiovascular risk (\%) & \\
At or above T7 (at risk) & 57 \\
Below T7 (not at risk) & 43 \\
\hline
\end{tabular}

Where applicable, data are presented as mean \pm standard deviation. Individuals were considered to be at risk of cardiovascular abnormalities (autonomic dysreflexia, orthostatic hypotension, and cardiac arrhythmia) if their self-reported injury level was at or above T7. of 231]) seated on a commode (47\% [109 of 232]), with some level of independence (61\% [140 of 229] completely independent or independent with the use of assistive devices). A multi-method approach to bowel care was reported. From a range of 11 possible bowel care interventions, most (43\% [103 of 240]) respondents used two or three methods in combination to complete their routine, $37 \%$ [ 88 of 240] used between four and ten methods in combination to complete their routine, and only $20 \%$ [ 49 of 240] used a single method. The most common interventions were digital stimulation (59\% [142 of 240]) and manual evacuation (45\% [109 of 240]). Duration of typical bowel care was greater than $30 \mathrm{~min}$ in $42 \%$ (96 of 228) and greater than $1 \mathrm{~h}$ in 24\% (55 of 228) of respondents. Only $63 \%$ (147 of 232) of respondents used diet/lifestyle management to enhance their bowel care.

Multivariate relationships between bowel management factors (unadjusted for potential confounds) can be seen in Table 3. A longer time to complete bowel care was associated with a more complicated bowel care routine (more approaches used; $p<0.001$ ). Individuals with a longer duration of injury were more likely to be older $(p<0.001)$ and not have made changes to their routine in recent years $(p<0.001$; Table 3$)$.

Most respondents (71\% [168 of 236]) had not modified any aspect of their bowel routine for more than 5 years.

Almost half (43\% [102 of 237]) of respondents were dissatisfied with their normal bowel management routine responding: "No, I'm dissatisfied" or "No, I'm very dissatisfied" with their current bowel care. Bowel care was also described as inflexible, with a majority ( $57 \%$ [128 of 224]) of respondents stating that their bowel care was either "Not flexible at all" or "Not very flexible." This inflexibility was associated with a decrease in overall QoL $(p<0.001)$.

Fecal incontinence was a concern for many respondents, with $33 \%$ (72 of 219) of respondents reporting incontinence at least once per month, including $16 \%$ (36 of 219) reporting incontinence every week (Table 2). Frequency of fecal incontinence was significantly higher in those with lower motor neuron neurogenic bowel disorder (46\% [11 of 24] experienced fecal incontinence at least once per week, $58 \%$ [14 of 24] at least once per month, and 33\% [8 of 24] less than once per month) than in those with upper motor neuron neurogenic bowel disorder (13\% [25 of 195] experienced fecal incontinence at least once per week, 30\% [58 of 195] at least once per month, and 47\% [91 of 195] less than once per month; $p=0.0033$ ). A high frequency of fecal incontinence was associated with a lower level of SCI ( $p<0.0083$; Table 3$)$. In addition, $18 \%$ (40 of 226) of individuals reported daily use of an incontinence pad or anal plug; there was a significant association between use of an incontinence pad or anal plug and the amount of fecal incontinence experienced $(p=0.008)$.

A large proportion of respondents (71\% [137 of 192]) reported intentionally restricting their fluid intake (reporting intentional fluid restriction rarely $22 \%$ [43 of 192], sometimes 29\% [55 of 192], and often 20\% [39 of 192]) because of concerns about their bladder management. Fluid restriction was associated with younger age $(p=0.0092)$ and more severe symptoms of $\mathrm{OH}(p=0.0146)$, $\mathrm{AD}(p=0.0003)$, and fatigue $(p=0.0178$; Table 3$)$.

\section{Cardiovascular symptoms during bowel care}

Autonomic dysreflexia and symptoms of cardiac arrhythmia. Although 67\% (163 of 245) of respondents had injuries at or above T7, placing them at higher risk of cardiovascular abnormalities, $72 \%$ (102 of 141) of these individuals reported 
Table 2. Bowel Care Management Practices

\begin{tabular}{|c|c|c|c|c|c|}
\hline & & $\%$ & & & $\%$ \\
\hline \multirow[t]{3}{*}{ Time of day } & Morning & 53 & \multirow{3}{*}{$\begin{array}{l}\text { Length of time using } \\
\text { current program }\end{array}$} & $<6$ months & 3 \\
\hline & Evening & 25 & & $<1$ year & 8 \\
\hline & Other & 23 & & $<5$ years & 18 \\
\hline \multirow[t]{7}{*}{ Total duration } & $0-5 \min$ & 9 & & $>5$ years & 71 \\
\hline & $6-10 \mathrm{~min}$ & 11 & \multirow[t]{4}{*}{ Assistance required } & Total assistance & 23 \\
\hline & 11-20 min & 20 & & Partial assistance & 16 \\
\hline & $21-30 \mathrm{~min}$ & 17 & & Use assistive devices & 30 \\
\hline & $31-60 \mathrm{~min}$ & 18 & & Completely independent & 31 \\
\hline & $61-90 \mathrm{~min}$ & 17 & \multirow[t]{2}{*}{ Flexibility } & Not flexible at all & 11 \\
\hline & $>90 \min$ & 7 & & Not very flexible & 46 \\
\hline \multirow[t]{7}{*}{ Frequency } & $<1$ per week & 1 & & Quite flexible & 29 \\
\hline & 1 per week & 3 & \multirow{5}{*}{ Position } & Very flexible & 13 \\
\hline & 2 per week & 7 & & Bed & 16 \\
\hline & $>2$ per week & 38 & & Commode & 47 \\
\hline & Daily & 38 & & Toilet seat & 18 \\
\hline & Twice-daily & 8 & & Other & 19 \\
\hline & 3 or more per day & 5 & \multirow[t]{5}{*}{ Fecal incontinence } & At least once daily & 5 \\
\hline \multirow{11}{*}{$\begin{array}{l}\text { Intervention used (multiple } \\
\text { responses possible) }\end{array}$} & Digital stimulation & 59 & & Once per week & 11 \\
\hline & Manual evacuation & 45 & & Once per month & 17 \\
\hline & Suppositories & 36 & & $<1$ per month & 45 \\
\hline & Abdominal massage & 31 & & Never & 18 \\
\hline & Straining/bearing down & 29 & \multirow[t]{7}{*}{ Use of pad or plug } & Every day & 18 \\
\hline & After drink/food & 29 & & At least once per week & 4 \\
\hline & Laxatives & 27 & & At least once per month & 3 \\
\hline & Stool softeners & 23 & & $<1$ per month & 8 \\
\hline & Other & 22 & & Never & 68 \\
\hline & Touching skin near anus & 10 & & & \\
\hline & Enema & 7 & & & \\
\hline
\end{tabular}

Data are expressed as the percentage of responses for each question. Most common responses are highlighted in bold.

experiencing $\mathrm{AD}$ at least once since their injury. Interestingly, $16 \%$ (35 of 213) of all respondents did not know whether they had ever experienced AD. Of those who did not know whether they had ever experienced AD, 54\% (19 of 35) had injury levels at or above T7, which makes them possible candidates for experiencing AD. Of these respondents $79 \%$ (15 of 19) experienced symptoms of AD during their routine bowel care.

Of those at risk for $\mathrm{AD}, 74 \%$ (123 of 163) reported at least one symptom of AD during their routine bowel care. Figure 1 shows the proportion and severity of AD symptoms reported during routine bowel care in those at risk for $\mathrm{AD}$. The most commonly reported symptoms of AD during bowel care were goosebumps (52\% [74 of 141]), spasticity (51\% [72 of 141]), flushing (49\% [70 of 142]), sweating (49\% [70 of 143]), general unwellness (43\% [61 of 142]), and headache (38\% [54 of 142]). Symptoms of cardiac arrhythmia (defined as heart palpitations, irregular heartbeats, or a feeling of fluttering in the chest) during bowel care were reported by $32 \%$ ( 45 of 141) of individuals with injuries at or above T7. Of those with injuries at or above T7 who reported symptoms of arrhythmia, their frequency of occurrence was described as occurring rarely (36\% [16 of 45]), monthly (21\% [9 of 45]), weekly (38\% [17 of 45]), and daily (7\% [3 of 45]). Typical severity of symptoms of arrhythmia during normal bowel care was reported as "mild" or "moderate."

We considered individuals with injuries at or above T7 who had at least one symptom of $\mathrm{AD}$ triggered during bowel care, to have $\mathrm{AD}(n=123)$, and the analysis of the following AD-specific questions were conducted on this subset of individuals. AD was reported to interfere with at least one activity by $68 \%$ ( 83 of 122) of re- spondents with $\mathrm{AD}$ and the impact was wide ranging. At least $50 \%$ of these respondents identified $\mathrm{AD}$ as interfering with social activities, activities of daily living, work, exercise, and sleep (Fig. 2A). Only $4 \%$ (4 of 110) of those with injuries at or above T7 reported deliberately provoking $\mathrm{AD}$ to boost sports performance. The majority of respondents who experienced $\mathrm{AD}$ identified a number of other stimuli that trigger these symptoms (Fig. 2B), most notably bladder care (89\%; [101 of 114]) and pain (75\%; [84 of 112]).

More severe symptoms of AD were associated with a younger age $(p=0.009)$, more complicated bowel care routine (more approaches used; $p<0.001)$, longer time to complete bowel care $(p=0.0018)$, greater frequency of fecal incontinence $(p<0.0075)$, and poorer QoL $(p<0.001$; Table 3$)$.

Orthostatic hypotension and fatigue. Fainting was reported by $39 \%$ ( 83 of 211 ) of all respondents, with recurrent episodes occurring in $33 \%$ (70 of 211 ); $10 \%$ (21 of 211) had fainted more than 8 times post-injury. Of respondents with injuries at or above $\mathrm{T} 7,93 \%$ (128 of 138) experienced at least one symptom of $\mathrm{OH}$. The most common symptoms were spasticity (80\% [109 of 137]), lightheadedness (75\% [104 of 138]), dizziness (75\% [103 of 138]), lethargy (72\% [99 of 138]), and nausea (59\% [81 of 138]; Fig. 3). The most common triggers of $\mathrm{OH}$ were posture changes $(63 \%$ [74 of 117]), bladder care (63\% [70 of 112]), and sitting (54\% [61 of 112]). $\mathrm{OH}$ was reported to interfere with a number of activities, including activities of daily living (39\% [43 of 111]), exercise (34\% [36 of 106]), work (32\% [30 of 94]), social activities (27\% [29 of $108])$, and rehabilitation (26\% [27 of 103]). 


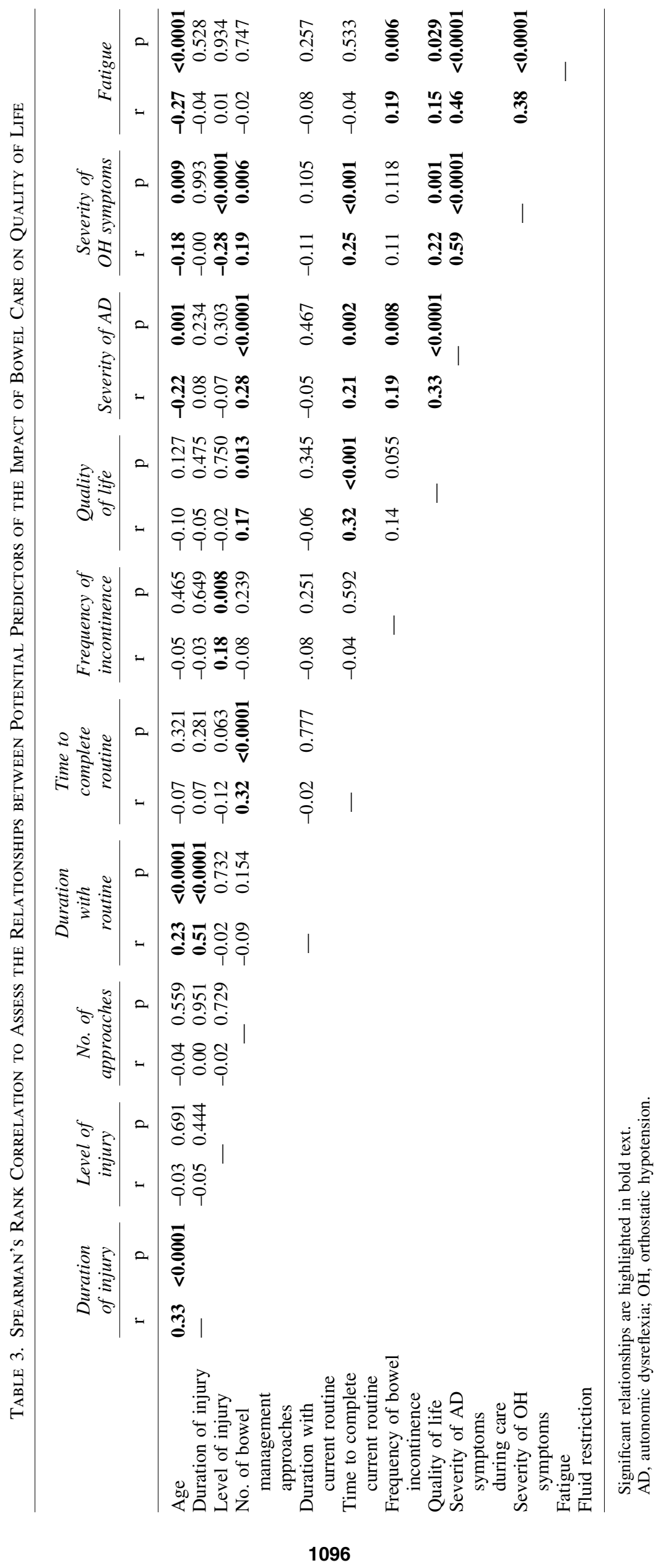




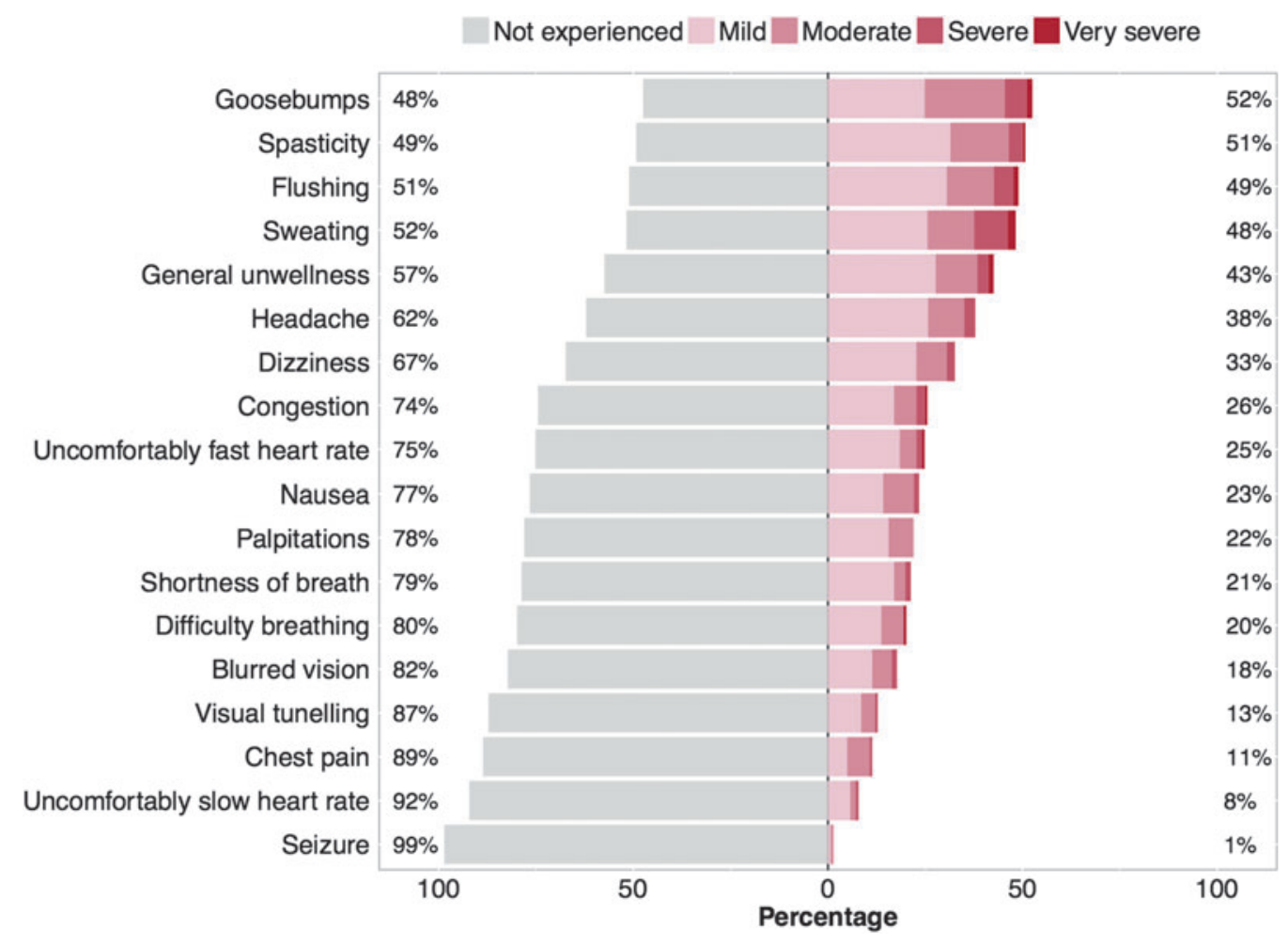

FIG. 1. Severity of symptoms of autonomic dysreflexia during bowel care in individuals with SCI considered at risk for cardiovascular dysfunction. Individuals were considered to be at risk for cardiovascular abnormalities if they had a self-reported lesion level at or above T7. Each symptom was classified according to its perceived severity. SCI, spinal cord injury.

Fatigue was commonly reported, with a mean fatigue score for all respondents $(n=214)$ of $3.6 \pm 0.2$. Higher levels of fatigue were associated with a younger age $(p=<0.0001)$, more frequent episodes of fecal incontinence $(p=0.0061)$, poorer $\operatorname{QoL}(p=0.0293)$, and more severe symptoms of $\mathrm{AD}(p=<0.0001)$ and $\mathrm{OH}(p<0.0001$; Table 3 ). There was a positive correlation between symptoms of $\mathrm{OH}$ and symptoms of $\mathrm{AD}$ in individuals with $\mathrm{SCI}$ at or above $\mathrm{T} 7$ $(p<0.0001 ; n=137)$. Individuals with more severe $\mathrm{OH}$ had higher level lesions $(p<0.0001)$, were more likely to be younger $(p=0.0092)$, more likely to fluid restrict because of bladder concerns $(p=0.0146)$, experienced more severe fatigue $(p<0.0001)$, and had a poorer QoL ( $p=0.001$; Table 3).

Multiple linear regression models $\left(r^{2}=0.72 ; p<0.0001 ; n=183\right)$ were constructed to establish the predictors of symptoms of $\mathrm{OH}$ for the sample as a whole when potential confounds were considered. Neurological level of injury $(\beta=-0.28 \pm 0.07 ; p=0.0001)$, severity of $\mathrm{AD}$ symptoms experienced $(\beta=0.58 \pm 0.04 ; p<0.0001)$, and fatigue $(\beta=0.71 \pm 0.18 ; p=0.0002)$ were the only significant predictors of severity of $\mathrm{OH}$ after adjustment for potential confounds.

\section{Impact of bowel management on quality of life}

Figure 4 compares the impact of different aspects of living with SCI on QoL on a scale from one (least effect) to ten (worst effect). The effect of SCI on bowel function was rated significantly worse than effects on sex $(p=0.024)$, bladder $(p<0.0001)$, pain $(p=0.013)$, spasticity $(p<0.0001)$, using a wheelchair $(p<0.0001)$, or skin issues $(p<0.0001)$. Impact of SCI on sexual function was greater than spasticity $(p<0.0001)$, wheelchair use $(p=0.0011)$, and skin integrity issues $(p<0.0001)$. Additional comparisons can be seen in Figure 3.
Negative impact of bowel management on QoL was apparent (Fig. 5), with 78\% (166 of 214) of respondents noting that bowel care was a problem for them (including $21 \%$ [46 of 214] who felt this was a major impact). Individuals reported that bowel care interfered with their social life, personal relationships, and prevented staying and working away from home.

When asked to qualitatively rank the impact of bowel care on QoL, 45\% (99 of 219) of respondents felt it had some impact and 29\% (63 of 219) felt it had a major impact on their QoL. QoL measures were internally validated by the significant relationships between this qualitative ranking of the impact of bowel care and the numerical scale reported $(p<0.001)$.

Potential predictors of the impact of bowel care on QoL and their inter-relationships can be seen in the multivariate correlation matrix in Table 3. An adverse impact of bowel care on QoL was associated with a more complicated bowel care routine (more approaches used) and a longer time to complete bowel care $(p<0.001)$. The relationship between a high frequency of fecal incontinence having an adverse impact on QoL did not quite achieve statistical significance $(p=0.055)$.

Multiple linear regression models were constructed to establish the predictors of QoL when potential confounds were considered (Table 4). Time to complete current bowel care routine $(p<0.001)$ and severity of AD symptoms during bowel care $(p=0.036)$ were the only significant predictors of QoL after adjustment for potential confounds. These components of bowel care were determined to be the primary factors associated with QoL. However, given the interrelationships between many of these variables, we constructed additional models to assess the secondary predictors of QoL. Level of injury ( $p=0.027$ ) and complexity of the care routine (number of bowel management approaches used; $p=0.001$ ) best predict the 
A

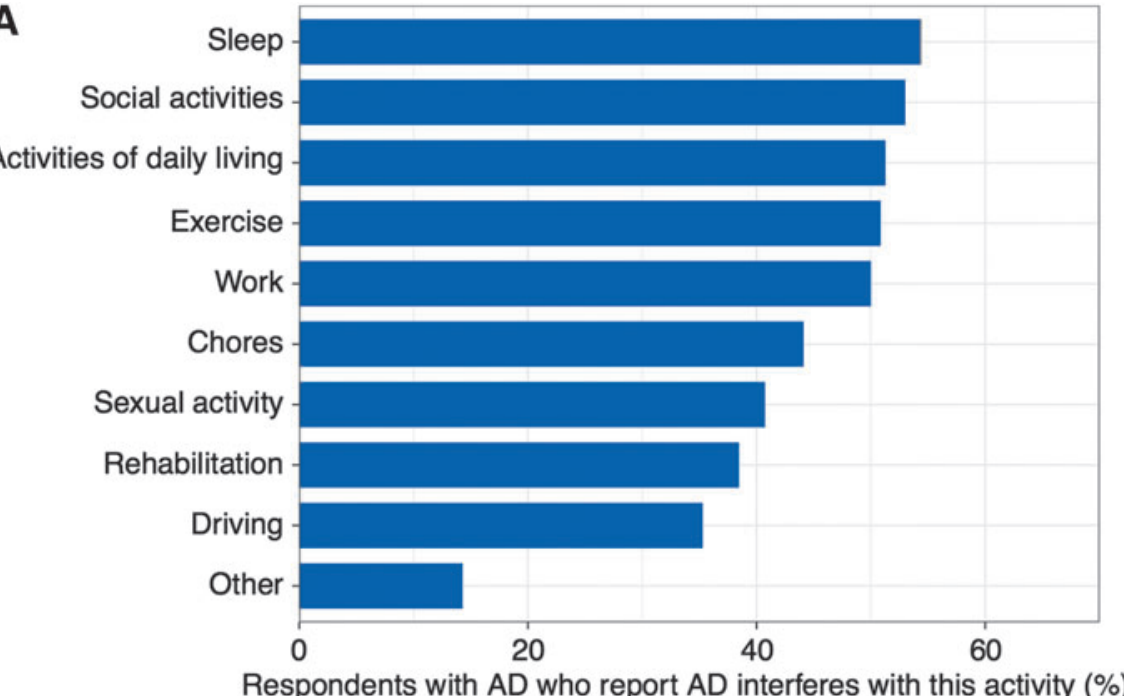

Respondents with $A D$ who report $A D$ interferes with this activity (\%)

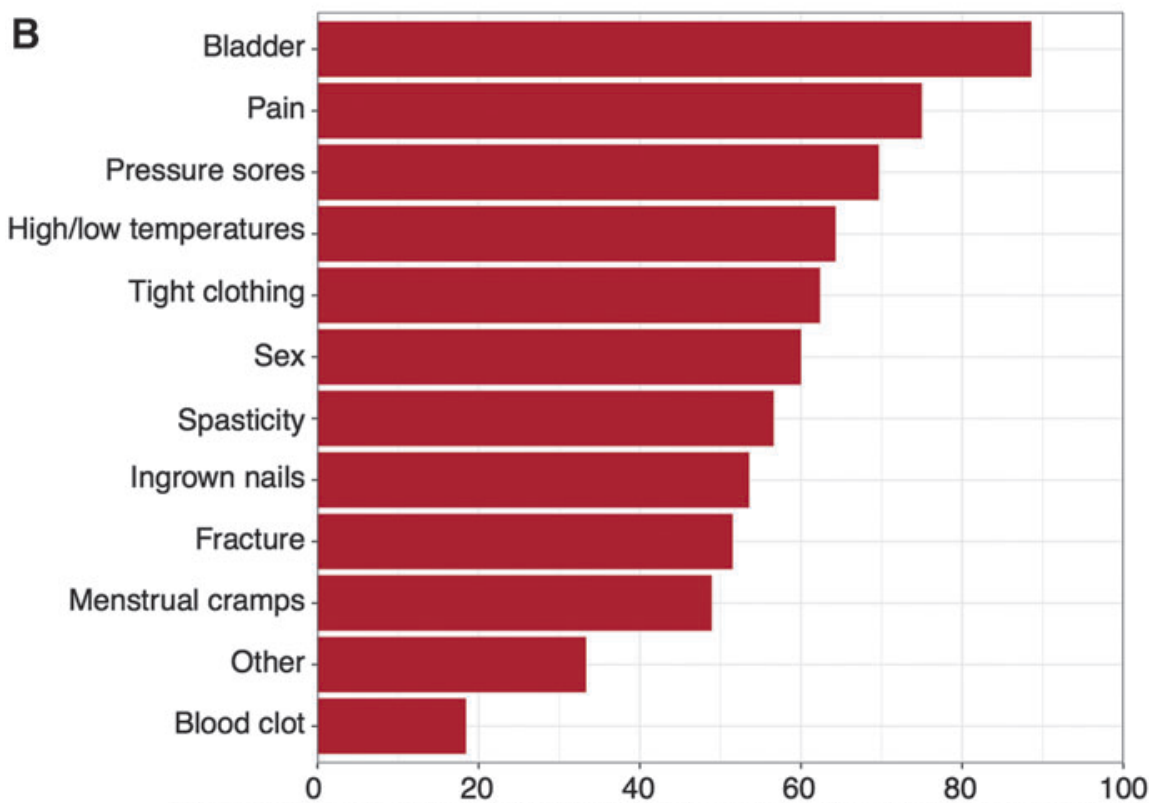

Other triggering stimuli in those with symptoms of $\mathrm{AD}$ during bowel care (\%)

FIG. 2. Influence of $\mathrm{AD}$ outside of bowel care. (A) Prevalence of autonomic dysreflexia interfering with common activities. (B) Additional stimuli that trigger autonomic dysreflexia symptoms in those with symptoms of autonomic dysreflexia during bowel care. $\mathrm{AD}$, autonomic dysreflexia.

time to complete current bowel care routines, whereas fatigue $(p<0.001)$ and number of bowel management approaches used $(p<0.001)$ best predict the severity of AD symptoms experienced during bowel care routines.

From this, we can consider a combined model for the impact of bowel care on QoL, with primary predictors of impaired QoL being a longer time to complete bowel care, and severe symptoms of AD. Secondary predictors of a severe impact of bowel care on QoL include a high level of injury, large number of bowel management approaches used, and severe fatigue (Fig. 6).

As a final consideration, we sought to consider potentially modifiable factors that are predictors of a severe impact of bowel care on QoL. We targeted the number of bowel management approaches as a factor that was potentially modifiable and strongly associated with both primary predictors of the impact of bowel care on QoL. Using
AIC model selection (Table 5), we identified the bowel management approaches that were most strongly associated with QoL (QoL was more impaired with use of enemas and suppositories than other methods), severity of AD symptoms (eating food or drink before bowel care was associated with more severe symptoms of AD than other methods), and time to complete bowel care (digital stimulation and suppositories increase time to complete bowel care, whereas normal defecation decreased the time to complete bowel care, compared to other methods).

\section{Discussion}

We have documented the profound impact of routine bowel management on QoL for a large sample of community-dwelling individuals with SCI. We also report the common bowel practices 


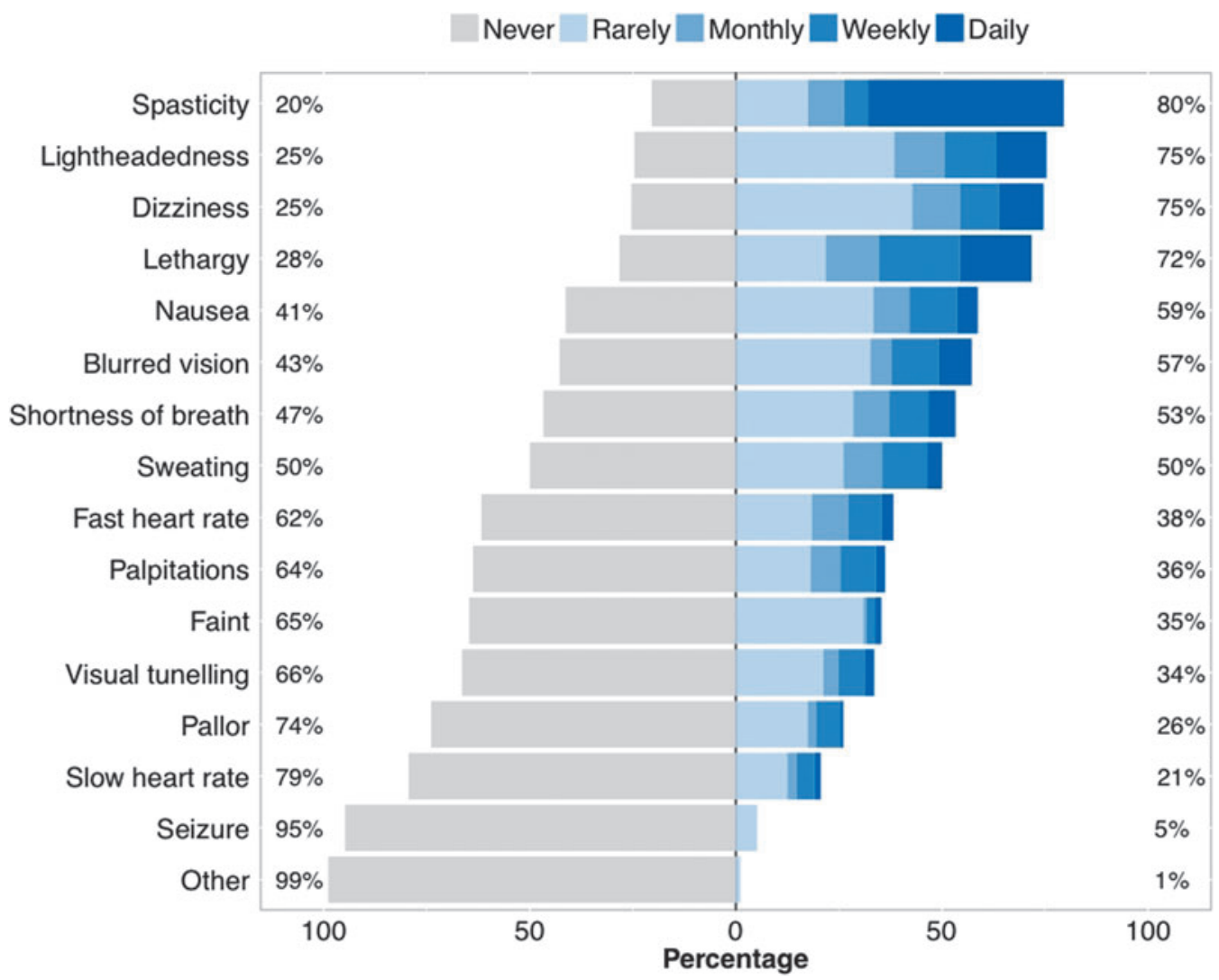

FIG. 3. Frequency of symptoms of orthostatic hypotension in individuals with SCI considered at risk for cardiovascular dysfunction. Individuals were considered to be at risk for cardiovascular abnormalities if they had a self-reported lesion level at or above T7. Each symptom was classified according to its perceived frequency. SCI, spinal cord injury.

used and the associations between bowel care and cardiovascular concerns. Our results suggest that there is significant opportunity to improve bowel management to minimize its impact on QoL. Further, a large proportion of individuals reported cardiovascular symptoms compatible with cardiac arrhythmia and AD during their routine bowel care. Although the long-term consequences of repeated $\mathrm{AD}$ remain unknown, minimizing its severity may be prudent for long-term cardiovascular health. ${ }^{21,43}$

\section{Bowel management after spinal cord injury}

Bowel management strategies described were multi-factorial and complicated. Respondents most commonly used a multi-step approach to bowel management, consistent with previous studies. 8 ,44 We have shown that an increase in the number of approaches used to complete bowel care had a negative impact on QoL through increases in the time taken to complete bowel care, as well as more severe symptoms of AD.

Fecal incontinence was a common concern and has been reported previously. ${ }^{8}$ As might be expected, ${ }^{9,45}$ this was particularly the case for those with a lower motor neuron bowel disorder and areflexive bowels. However, despite concerns about incontinence, it was no longer significantly associated with QoL when potential confounding factors were considered. This does not necessarily mean that minimizing fecal incontinence is not an important target for improvement of QoL; rather that these relationships are complex and not independent of other factors such as age, duration of injury, and level of injury.
Fatigue was a common concern, with high levels of fatigue reported, particularly in younger individuals. Fatigue was strongly associated with severity of $\mathrm{OH}$, presumably through hypotension and cerebral hypoperfusion. ${ }^{46-51}$ Surprisingly, in the specific context of bowel care, fatigue was a secondary predictor of QoL through its association with severity of AD. This might reflect the association between $\mathrm{AD}$ and $\mathrm{OH},{ }^{5}$ which often manifests symptomatically as fatigue. ${ }^{52}$

In our sample, $71 \%$ of individuals reported intentional fluid restriction because of bladder management concerns, with $49 \%$ of individuals using fluid restriction "sometimes or often." Younger individuals were more likely to use fluid restriction. We considered whether this might impact the time to complete bowel care or severity of symptoms of $\mathrm{AD}$, based on the theoretical risk of worsening constipation. We found that fluid restriction was associated with the severity of $\mathrm{AD}$, but not the time to complete bowel care. In addition, fluid restriction was strongly related to the severity of $\mathrm{OH}$ and fatigue reported, presumably through hypovolemia. ${ }^{5}$ These data highlight the need to consider a holistic approach to the management of bladder and bowel care post-SCI, enhancing bladder care to prevent fluid restriction and optimizing bowel care to reduce symptoms of cardiovascular dysfunction.

Current clinical guidelines suggest that a bowel routine is problematic if it exceeds $1 \mathrm{~h}$ in duration. ${ }^{10}$ According to this definition, one quarter of respondents had problematic routines (a further $18 \%$ had typical bowel routines that exceeded $30 \mathrm{~min}$ ). Given the strong association between the duration of bowel care routines and QoL, these data suggest an urgent need to investigate 


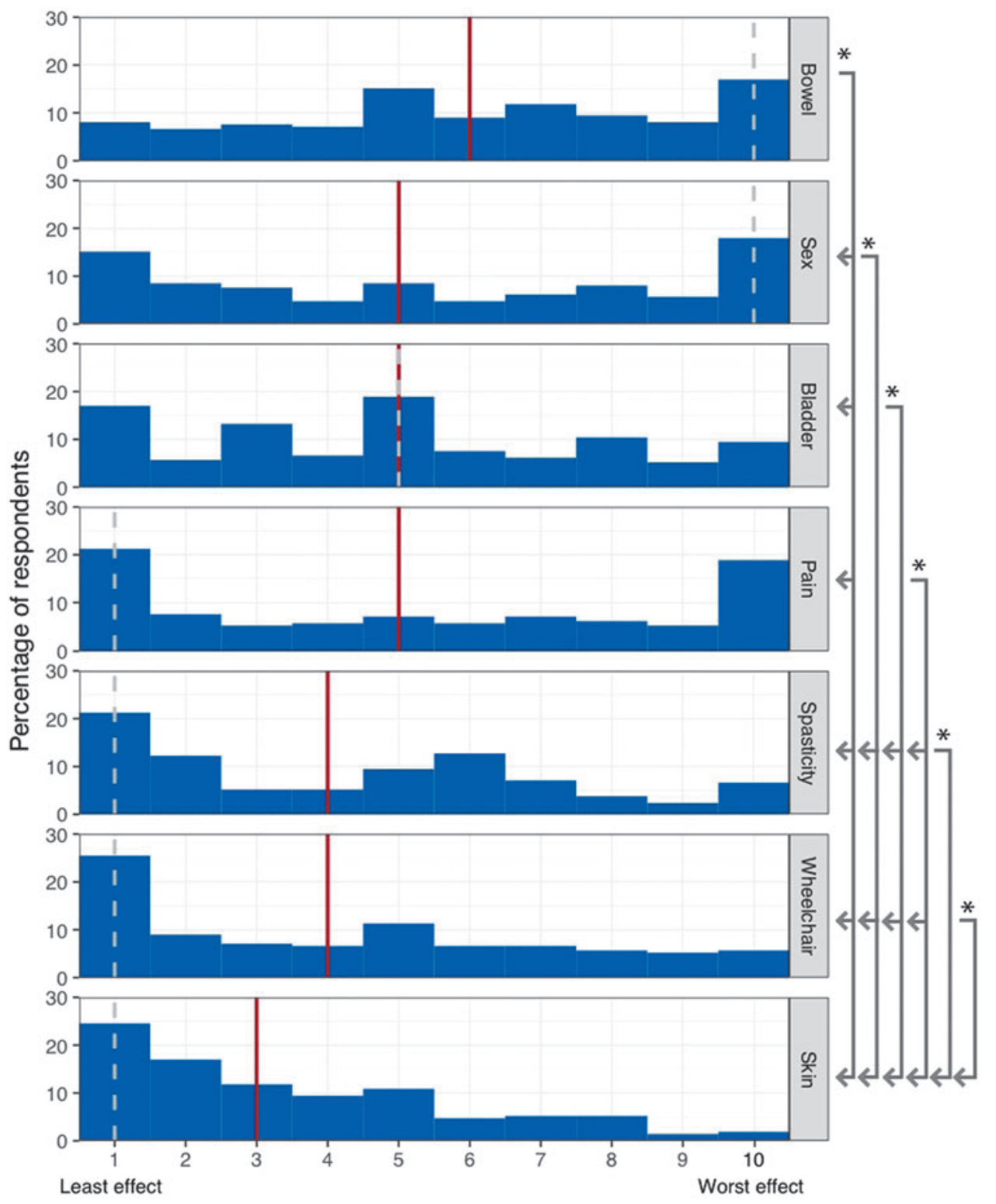

Impact on quality of life compared to other aspects of spinal cord injury

FIG. 4. Impact of different aspects of living with spinal cord injury on quality of life. Respondents were asked to rate each aspect of living with spinal cord injury from 1 (Least effect) to 10 (Worst effect). Solid red line indicates group median; dotted gray line indicates group mode. Asterisk indicates significant difference between indicated aspects $(p<0.05)$.

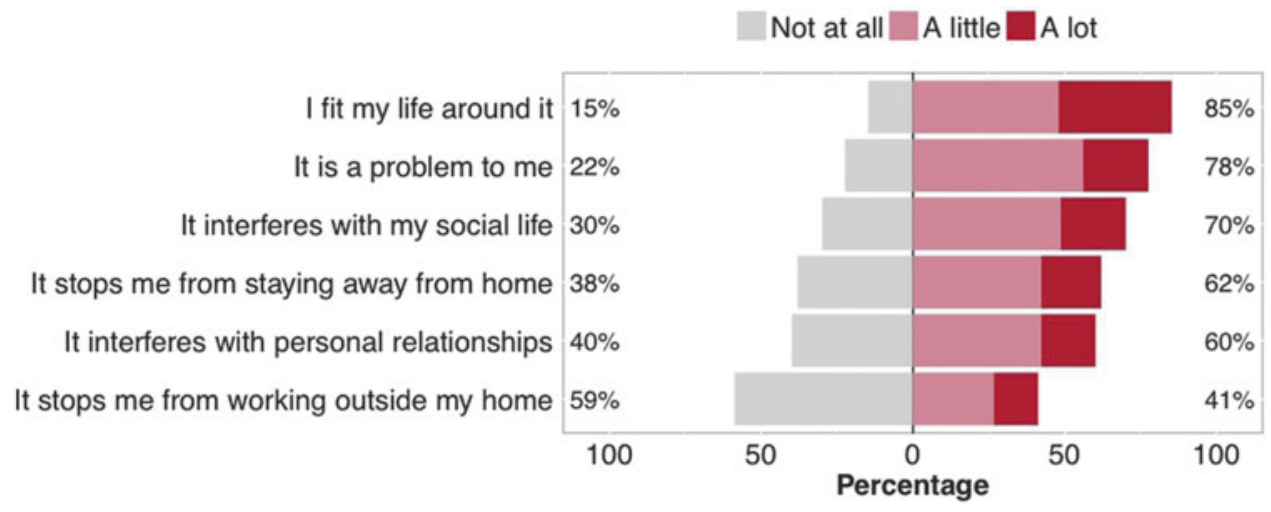

FIG. 5. Impact of bowel management on respondents' life and activities. 
Table 4. Primary and Secondary Predictors of the Impact of Bowel Care on Quality of Life

\begin{tabular}{|c|c|c|}
\hline & \multicolumn{2}{|c|}{$\begin{array}{c}\mathrm{r}=0.22 ; \mathrm{p}<0.001 \\
\mathrm{n}=170\end{array}$} \\
\hline & $\beta(S E)$ & $\mathrm{p}$ \\
\hline \multicolumn{3}{|l|}{ Primary predictors of quality of life } \\
\hline Duration of injury & $0.023(0.02)$ & 0.753 \\
\hline Level of injury & $0.021(0.03)$ & 0.779 \\
\hline $\begin{array}{l}\text { No. of bowel management } \\
\text { approaches used }\end{array}$ & $0.103(0.12)$ & 0.182 \\
\hline Duration with current routine & $0.017(0.33)$ & 0.823 \\
\hline Time to complete current routine & $0.308(0.12)$ & $<0.001$ \\
\hline Frequency of bowel incontinence & $0.064(0.14)$ & 0.399 \\
\hline $\begin{array}{l}\text { Severity of AD symptoms } \\
\text { during routine }\end{array}$ & $0.185(2.31)$ & 0.036 \\
\hline Fatigue & $0.119(0.08)$ & 0.157 \\
\hline Amount of fluid restriction & $-0.078(0.19)$ & 0.287 \\
\hline \multirow{2}{*}{$\begin{array}{l}\text { Predictors of time to complete } \\
\text { current routine }\end{array}$} & \multicolumn{2}{|c|}{$\begin{array}{c}\mathrm{r}=0.12 ; \mathrm{p}=0.0018 \\
\mathrm{n}=176\end{array}$} \\
\hline & $\beta(S E)$ & $\mathrm{p}$ \\
\hline \multicolumn{3}{|l|}{ Secondary predictors of quality of life } \\
\hline Level of injury & $-0.165(0.019)$ & 0.027 \\
\hline $\begin{array}{l}\text { No. of bowel management } \\
\text { approaches used }\end{array}$ & $0.259(0.073)$ & 0.001 \\
\hline Duration with current routine & $-0.059(0.211)$ & 0.448 \\
\hline Frequency of bowel incontinence & $-0.082(0.091)$ & 0.293 \\
\hline Fatigue & $0.023(0.045)$ & 0.763 \\
\hline Amount of fluid restriction & $-0.108(0.117)$ & 0.152 \\
\hline Duration of injury & $0.033(0.010)$ & 0.670 \\
\hline \multirow{2}{*}{$\begin{array}{l}\text { Predictors of severity of } A D \\
\text { symptoms during routine }\end{array}$} & \multicolumn{2}{|c|}{$\begin{array}{c}\mathrm{r}=0.33 ; \mathrm{p}<0.001 \\
\mathrm{n}=176\end{array}$} \\
\hline & $\beta(S E)$ & $\mathrm{p}$ \\
\hline Level of injury & $-0.113(0.001)$ & 0.083 \\
\hline $\begin{array}{l}\text { No. of bowel management } \\
\text { approaches used }\end{array}$ & $0.264(0.004)$ & $<0.001$ \\
\hline Duration with current routine & $-0.029(0.011)$ & 0.674 \\
\hline Frequency of bowel incontinence & $0.107(0.005)$ & 0.115 \\
\hline Fatigue & $0.428(0.198)$ & $<0.001$ \\
\hline Amount of fluid restriction & $0.071(0.006)$ & 0.277 \\
\hline Duration of injury & $0.041(0.001)$ & 0.541 \\
\hline
\end{tabular}

Significant relationships are highlighted in bold. Relationships are expressed as $\beta$ (standard error [SE]).

$\mathrm{AD}$, autonomic dysreflexia.

and implement bowel management approaches that decrease the time to complete routine bowel care. In our analyses, the bowel management approaches most associated with long bowel care routines were use of suppositories and digital stimulation; in those able to use normal defecation (bearing down), the time to complete care was shorter.

\section{Associations between bowel care and cardiovascular dysfunction}

Of those most at risk for experiencing cardiovascular dysfunction post-SCI (defined as those with lesions at or above T7), 74\% reported at least one symptom of AD during bowel care and $32 \%$ described symptoms compatible with cardiac arrhythmia. The most common symptoms reported were goosebumps, spasticity, sweating, flushing, a feeling of unwellness, and headache-very typical symptoms associated with $\mathrm{AD} . .^{30,53-55}$ In those who experienced symptoms of $\mathrm{AD}$ during bowel care, episodes of $\mathrm{AD}$ were also commonly triggered during other activities of daily living, most notably by bladder care and pain, suggesting that most individuals who are susceptible to AD are experiencing multiple episodes per day. Many of these stimuli are unavoidable, and bowel care in particular is a stimulus of long duration. Therefore, it is likely that improvement of AD is a target not only for improving the impact of bowel care on QoL, but also for improved management of cardiovascular sequalae. The long-term effects of repeated bouts of $\mathrm{AD}$ have yet to be fully elucidated, but there is evidence of altered vascular function in animal models of $\mathrm{AD},{ }^{21}$ and there are case reports of cerebrovascular accidents and hemorrhage secondary to AD. ${ }^{26,27,53}$ These hypertensive episodes often occur in conjunction with constitutional hypotension and $\mathrm{OH}$, as noted by survey respondents, and the impact of these large swings in blood pressure may challenge the integrity of the endothelium, vessel wall, and cardiac function. ${ }^{56}$

Although we were primarily interested in the relationships between $\mathrm{AD}$ and bowel care, it was interesting that $\mathrm{AD}$ was reported to interfere with numerous other common activities, including sleep, exercise, normal daily activities, and sexual function. Many of these triggers are well known ${ }^{53,55}$ and underscore the need to find effective management strategies for AD. ${ }^{55}$ However, the association between $\mathrm{AD}$ interfering with sleep has not been reported previously, to our knowledge. This may seem surprising, because we do not think of many triggers for AD being present during sleep. However, considering that most respondents reported conducting their bowel care first thing in the morning or in the evening, it is possible the colon filling initiates $\mathrm{AD}$ ahead of morning bowel care, or that evening bowel care triggers symptoms of $\mathrm{AD}$ that continue unabated-even once the offending stimulus has been removed. ${ }^{57}$ Further, bladder triggers for AD may also be peaking in the night as the bladder progressively fills. Urine production is also higher in the night than the day in individuals with high-level lesions, which could further exacerbate the nocturnal bladder stimulus. ${ }^{58}$ Together, these triggers could combine to exacerbate AD and interfere with sleep. Disruption of sleep is particularly concerning among individuals with high-level SCI who experience $\mathrm{AD}$ because many already experience profound fatigue related to low resting cerebral blood flow during the day, ${ }^{46-51}$ and poor sleep further exacerbates fatigue, difficulty concentrating, and excessive daytime sleepiness. ${ }^{52}$

Most individuals (84\%) were aware of AD and whether they had experienced it. This suggests that education strategies in this area have largely been effective. However, a number of respondents with lesion levels placing them at risk for AD did not know if they had it, despite describing symptoms of AD during their bowel care. This suggests there is still some room for improvement in education about the risk profile, identifying signs and symptoms of $\mathrm{AD}$, and when to seek assistance for AD management. More generally, although $\mathrm{AD}$ awareness was high in this sample, $\mathrm{AD}$ was described as impacting QoL and interfering with activities of daily living, suggesting that education and resources concerning the management of $\mathrm{AD}$ are a priority for those living with SCI. Those who were particularly likely to experience severe symptoms of $\mathrm{AD}$ were those who were younger, with more complicated bowel care routines, and those for whom bowel care takes a long time. It is probable that the longer duration and magnitude of the afferent stimulus for $\mathrm{AD}$ 
during more complex and longer duration routines underlies the more severe symptoms of $\mathrm{AD}$, highlighting the need to ameliorate symptoms in these individuals, either pharmacologically or behaviorally, through reductions in the time to complete bowel care and decreases in the number of stimuli used.

In terms of reducing the severity of $\mathrm{AD}$ pharmacologically, one approach has been to mitigate the afferent stimulus using anesthetic lubricants during bowel care. However, guidelines regarding use of topical anesthesia to minimize AD during known triggers, such as during bowel care, are conflicting, ${ }^{7,10,59,60}$ and this is noted in the SCI community, with uncertainty about the different techniques and strategies to manage AD when performing bowel care at home. ${ }^{60,61}$ Clearer recommendations and additional education are warranted.

Symptoms of $\mathrm{OH}$, including fainting, lightheadedness, dizziness, and lethargy, were also reported by a significant proportion of respondents. Triggers for these symptoms included positional changes and sitting. These symptoms can further compound the challenges that individuals with SCI face in their daily lives, work, and leisure activities and were strongly associated with fatigue. ${ }^{62,63}$ There is mounting evidence that hypotension is not a benign condition, and that hypotension and associated cerebral hypoperfusion should be avoided, including documented attention deficits and other mild cognitive impairments in hypotensive individuals with SCI. ${ }^{63}$ Therefore, there is a strong rationale to increase education about hypotension, symptoms, and positional counter maneuvers and, potentially, to encourage self-monitoring of blood pressure at home. ${ }^{63,64}$ These measures should be targeted to those most at riskindividuals with high-level lesions and younger individuals with SCI. In particular, given that $71 \%$ of individuals reported intentionally restricting their fluid intake because of concerns with bladder management, the holistic management of bladder and bowel function and the importance of adequate fluid intake should be stressed.

\section{Quality of life}

It was clear that a significant proportion of individuals (78\%) felt that their current bowel management was a problem to them. When compared to other aspects of SCI, difficulties with bowel management were more frequently rated as being one of the worst effects of living with SCI. Impaired sexual function and bladder concerns were also ranked as severe consequences of injury, highlighting the impact of autonomic dysfunctions in general on QoL post-SCI. Living with chronic pain was also ranked as a significant concern. However, aspects such as skin care, spasticity, and use of a wheelchair were rated as having much lower effects on individuals' lives. This suggests that these aspects have been well managed within this population - they can be areas of significant importance and concern when they are problematic. ${ }^{65,66}$

Some of the reasons for the major effect of bowel management on QoL were highlighted by respondents, with the majority commenting that there was little or no flexibility in their bowel management routine $(57 \%)$, meaning that they had to fit their life around their bowel care (85\%). Bowel management was identified to interfere with social activities $(70 \%)$, personal relationships $(60 \%)$, as well as staying (62\%) and working (41\%) away from home. In this study, we did not ask questions that would allow us to examine the particular domains of QoL that were affected; however, a negative impact on many domains of QoL (social, emotional, physical, etc.) was evident in the survey data. Future studies should address this important consideration.

What is perhaps most surprising is that, despite the clear concerns raised by the majority of survey respondents about their bo- wel care, most $(71 \%)$ have not made any changes to their bowel routine for at least 5 years. These data highlight gaps in bowel management education and raise questions about current guidelines for bowel managent, and where individuals with SCI and their caregivers obtain information about this topic. Formal guidelines are available from agencies such as the Paralyzed Veterans of America, ${ }^{10,67}$ but these publications can become dated quickly as new medications and equipment arrive on the market. Web-based education material and videos (such as http://www.sci-u.ca/) serve the need to remain current and provide engaging and accessible resources. ${ }^{68}$ Activity on online SCI resources suggest many people also share and obtain information on bowel care from individuals with SCI and their caregivers. ${ }^{60}$ A combination of formal accessible and up-to-date guidelines, with real-time support from healthcare providers and trained peer counselers, when necessary (either online, over the phone, or in person), would seem an ideal combination of resources to provide advice and support for bowel care. This kind of approach has been sucessful in managing other SCI complications, including pressure ulcers, depression, and hand function. ${ }^{69,70}$ Ongoing support and education is essential for continued effective bowel care management, especially as changes in gastrointestinal function occur over time post-injury and with aging, demanding ongoing adaptations to routine management protocols.

Our findings are consistent with past studies that have also found a decrease in QoL attributed to bowel management. ${ }^{8,44}$ However, our analyses discovered primary and secondary predictors not yet mentioned in the literature. We have identified a framework (Fig. 6), to assist community stakeholders, policy makers, and healthcare providers in identifying the key targets for enhancement (of the numerous potential modifiable factors) in order to improve bowel care and therefore QoL for individuals living with SCI. The primary predictors of a severe impact of bowel care on QoL after adjustment for confounds, were a longer time to complete bowel care and severe symptoms of AD. Secondary predictors were highlevel injuries, more complicated bowel care routines, and more severe fatigue. As noted above, optimizing bladder care to limit fluid restriction and so improve fatigue, and implementing strategies to reduce both the time taken to complete bowel care and the number of approaches need to complete bowel care, would be key targets to improve QoL and reduce the severity of $\mathrm{AD}$. Adjunct therapy to further ameliorate symptoms of $\mathrm{AD}$ may also be of benefit.

\section{Strengths and limitations}

Our study displays several strengths. We were able gather extensive information on a range of bowel care and related concerns from a large group of community-dwelling individuals living with SCI, with a range of ages, duration of injury, and level and severity of lesion. Survey completion rates were also high (73\%).

Despite having achieved an array of respondents from different locations, the majority of our respondents were from Canada (55\%). This may have had an effect, given that the rehabilitation and localization of resources in this area could lead to homogeneous approaches to bowel management. However, we were able to collect information outside of the Canadian perspective, and the trends discussed were consistent through all respondents regardless of location.

As with any survey, it is possible that our sample does not represent all individuals with SCI. In particular, we likely have a higher representation of individuals for whom issues around bowel 


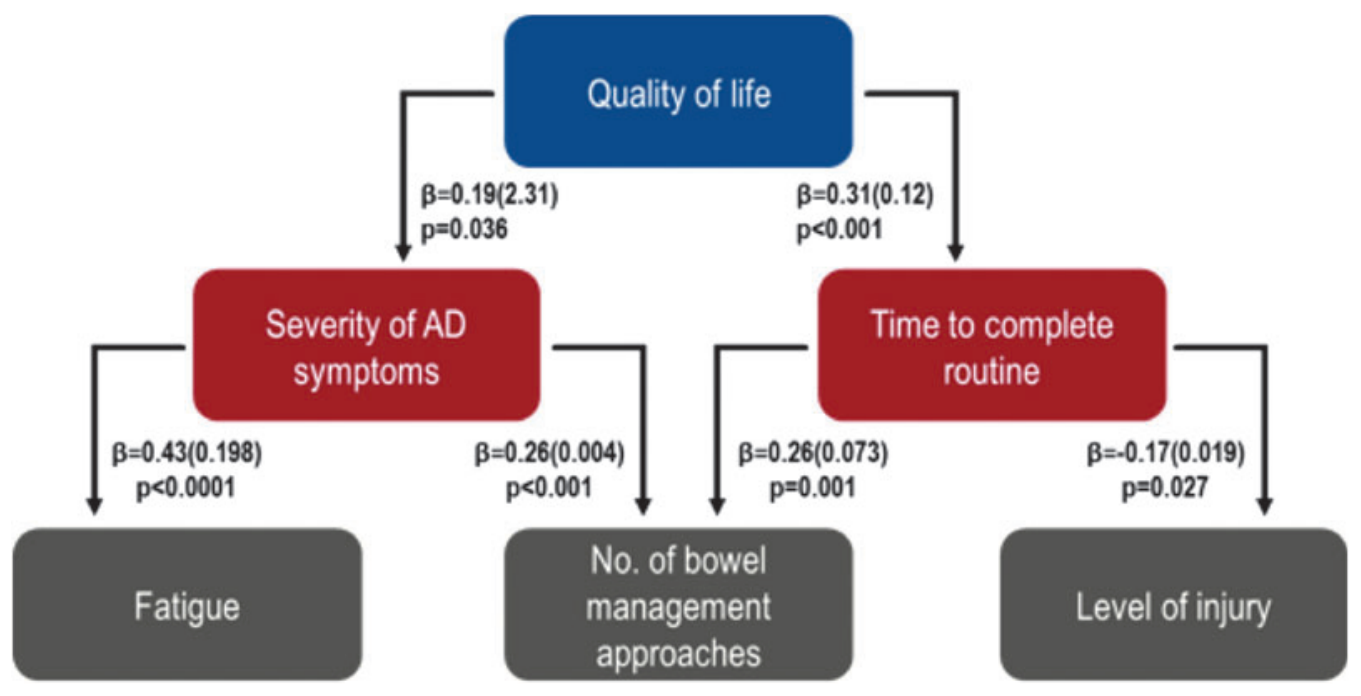

FIG. 6. Primary and secondary factors influencing quality of life. Relationships are expressed as $\beta$ (standard error). Primary predictors are shown in red boxes and secondary predictors in gray boxes. AD, autonomic dysreflexia.

care resonate, and therefore who are not happy with their bowel management routine. These individuals may be more likely to share their experiences and seek out information about alternative bowel care management options. However, this population would not necessarily be more prone to cardiovascular concerns, so the cardiovascular findings are more likely to be robust and representative of the general SCI population.

When considering those at high risk for cardiovascular concerns post-SCI, we used a self-reported lesion level cutoff of $\mathrm{T} 7$ and above to designate high risk. This is a conservative cutoff-more typically $\mathrm{T} 5$ or $\mathrm{T} 6$ would be used to discriminate $\mathrm{e}^{53,55}$; there is a precedent for this approach in self-report studies. ${ }^{8}$ We chose this lower cutoff because these data are based on self-reported spinal levels in the mid-thoracic region, which is hard to test from a motor perspective, and allowing for some ambiguity in participants' knowledge of their sensory level of injury. However, the potential inclusion of some individuals without severe injury to cardiovascular autonomic nerves in the high-risk group (either because of the conservative cut-off criterion or because of the known possibility for a disconnect between motor, sensory, and autonomic severity of injury) may have underestimated the true impact of cardiovascular autonomic concerns in this study.

Finally, although we used survey items that had been developed and used previously in a SCI cohort, we acknowledge that many of these tools have not undergone rigorous psychometric evaluation.

\section{Conclusions}

Bowel care and symptoms of cardiovascular dysfunction are a significant concern for individuals living with SCI, with a profound impact on QoL. Despite these concerns, most individuals are not making changes to their bowel care, highlighting gaps in bowel management education, and raising questions about current guidelines for bowel management. These data demonstrate the need for better information and resources about this topic for individuals with SCI and their caregivers.

Table 5. Akaike Information Criterion (AIC) Variable Importance Score for Management Approaches to Bowel Care in Relation to Quality of Life, Severity of AD Symptoms, and Time to Complete the Bowel Routine

\begin{tabular}{|c|c|c|c|c|c|c|c|c|c|}
\hline \multirow[b]{3}{*}{ Bowel management approaches } & \multicolumn{9}{|c|}{ AIC variable importance score with parameter estimates $( \pm S D)$} \\
\hline & \multicolumn{3}{|c|}{ Quality of life } & \multicolumn{3}{|c|}{ Severity of $A D$ symptoms } & \multicolumn{3}{|c|}{ Time to complete routine } \\
\hline & $A I C$ & Estimate & $S D$ & $A I C$ & Estimate & $S D$ & $A I C$ & Estimate & $S D$ \\
\hline Manual evacuation & 0.29 & 0.115 & $(0.25)$ & 0.20 & -0.064 & $(0.35)$ & 0.24 & -0.005 & $(0.06)$ \\
\hline Laxatives & 0.24 & 0.016 & $(0.15)$ & 0.19 & 0.051 & $(0.36)$ & 0.25 & -0.013 & $(0.08)$ \\
\hline Stool softeners & 0.23 & -0.037 & $(0.16)$ & 0.20 & 0.066 & $(0.38)$ & 0.25 & 0.012 & $(0.07)$ \\
\hline Massaging or rubbing abdomen & 0.41 & 0.258 & $(0.41)$ & 0.43 & 0.898 & $(1.36)$ & 0.29 & 0.047 & $(0.11)$ \\
\hline Touching the skin around the anus & 0.37 & -0.301 & $(0.52)$ & 0.68 & 2.839 & $(2.70)$ & 0.42 & -0.180 & $(0.28)$ \\
\hline Food/drink before management & 0.24 & -0.036 & $(0.16)$ & 0.81 & 2.839 & (2.01) & 0.51 & 0.192 & $(0.25)$ \\
\hline Enemas & 0.90 & 2.122 & (1.11) & 0.46 & 1.860 & $(2.68)$ & 0.75 & 0.663 & $(0.53)$ \\
\hline Normal defecation & 0.38 & -0.241 & $(0.41)$ & 0.20 & -0.058 & $(0.39)$ & 0.91 & -0.606 & (0.29) \\
\hline Digital stimulation & 0.31 & 0.128 & $(0.27)$ & 0.24 & -0.211 & $(0.55)$ & 1.0 & 0.972 & $(0.23)$ \\
\hline Suppositories & 0.91 & 1.071 & $(0.55)$ & 0.31 & 0.423 & $(0.82)$ & 1.0 & 2.129 & $(0.22)$ \\
\hline
\end{tabular}

A score $\geq 0.8$ indicates factors with high variable importance (highlighted in bold text). Parameter estimates and the standard deviation (SD) of the estimate are provided.

$\mathrm{AD}$, autonomic dysreflexia; SD, standard deviation. 


\section{Acknowledgments}

We are grateful to the Rick Hansen Institute, International Collaboration On Repair Discoveries, and Spinal Cord Injury British Columbia for helping to raise awareness of this survey. This work was funded by the Craig H. Neilsen Foundation. Dr. Claydon received salary support through a New Investigator Award from the Heart and Stroke Foundation of Canada during the period of this work.

\section{Author Disclosure Statement}

No competing financial interests exist.

\section{References}

1. Adams, M., and Cavanagh, J. (2004). International Campaign for Cures of Spinal Cord Injury Paralysis (ICCP): another step forward for spinal cord injury research. Spinal Cord 42, 273-280.

2. Singh, A., Tetreault, L., Kalsi-Ryan, S., Nouri, A., and Fehlings, M.G. (2014). Global prevalence and incidence of traumatic spinal cord injury. Clin. Epidemiol. 6, 309-331.

3. Karlsson, A.-K. (2006). Autonomic dysfunction in spinal cord injury: clinical presentation of symptoms and signs. Prog. Brain Res. 152, 1-8.

4. Anderson, K. (2006). Autonomic Dysfunction After Spinal Cord Injury. Elsevier: Amsterdam, pps. xi-xii.

5. Claydon, V.E., Steeves, J.D., and Krassioukov, A.V. (2006). Orthostatic hypotension following spinal cord injury: understanding clinical pathophysiology. Spinal Cord 44, 341-351.

6. Anderson, K.D. (2004). Targeting recovery: priorities of the spinal cord-injured population. J. Neurotrauma 21, 1371-1383.

7. Stiens, S.A., Biener Bergman, S., and Goetz, L.L. (1997). Neurogenic bowel dysfunction after spinal cord injury: clinical evaluation and rehabilitative management. Arch. Phys. Med. Rehabil. 78, 3 Suppl., S86-S102.

8. Coggrave, M., Norton, C., and Wilson-Barnett, J. (2009). Management of neurogenic bowel dysfunction in the community after spinal cord injury: a postal survey in the United Kingdom. Spinal Cord 47, 323-333.

9. Krassioukov, A., Eng, J., Claxton, G., Sakakibara, B., and Shum, S. (2010). Neurogenic bowel management after spinal cord injury: a systematic review of the evidence. Spinal Cord 48, 718-733.

10. Consortium for Spinal Cord Medicine. (1998). Neurogenic Bowel Management in Adults with Spinal Cord Injury. Consortium for Spinal Cord Medicine: Washington, DC.

11. Chung, E.A., and Emmanuel, A.V. (2006). Gastrointestinal symptoms related to autonomic dysfunction following spinal cord injury. Prog. Brain Res. 152, 317-333.

12. Kirshblum, S.C., Gulati, M., O'Connor, K.C., and Voorman, S.J. (1998). Bowel care practices in chronic spinal cord injury patients. Arch. Phys. Med. Rehabil. 79, 20-23.

13. Claydon, V.E., and Krassioukov, A.V. (2007). Clinical correlates of frequency analyses of cardiovascular control after spinal cord injury. Am. J. Physiol. 294, H668-H678.

14. Claydon, V.E., Elliott, S.L., Sheel, A.W., and Krassioukov, A. (2006). Cardiovascular responses to vibrostimulation for sperm retrieval in men with spinal cord injury. J. Spinal Cord Med. 29, 207-216.

15. Krassioukov, A., and Claydon, V.E. (2006). The clinical problems in cardiovascular control following spinal cord injury: an overview. Prog. Brain Res. 152, 223-229.

16. Inskip, J.A., Ramer, L.M., Ramer, M.S., Krassioukov, A.V., and Claydon, V.E. (2012). Spectral Analyses of Cardiovascular Control in Rodents with Spinal Cord Injury. J. Neurotrauma 29, 1638-1649.

17. Ravensbergen, H.J., Walsh, M.L., Krassioukov, A.V., and Claydon, V.E. (2012). Electrocardiogram-based predictors for arrhythmia after spinal cord injury. Clin. Auton. Res. 22, 265-273.

18. Garshick, E., Kelley, A., Cohen, S., Garrison, A., Tun, C., Gagnon, D., and Brown, R. (2005). A prospective assessment of mortality in chronic spinal cord injury. Spinal Cord 43, 408-416.

19. Myers, J., Lee, M., and Kiratli, J. (2007). Cardiovascular disease in spinal cord injury. Am. J. Phys. Med. Rehabil. 86, 142-152.

20. Cragg, J.J., Noonan, V.K., Krassioukov, A.V., and Borisoff, J.F. (2013). Cardiovascular disease and spinal cord injury: results from a national population health survey. Neurology 81, 723-728.
21. Alan, N., Ramer, L.M., Inskip, J.A., Golbidi, S., Ramer, M.S., Laher, I., and Krassioukov, A.V. (2010). Recurrent autonomic dysreflexia exacerbates vascular dysfunction after spinal cord injury. Spine J. 10, $1108-1117$.

22. Kursh, E.D., Freehafer, A., and Persky, L. (1977). Complications of autonomic dysreflexia. J. Urol. 118, 70-72.

23. Lindan, R., Joiner, E., Freehafer, A.A., and Hazel, C. (1980). Incidence and clinical features of autonomic dysreflexia in patients with spinal cord injury. Paraplegia 18, 285-292.

24. Yarkony, G.M., Katz, R.T., and Wu, Y.C. (1986). Seizures secondary to autonomic dysreflexia. Arch. Phys. Med. Rehabil. 67, 834-835.

25. Colachis, S.C. III, and Clinchot, D.M. (1997). Autonomic hyperreflexia associated with recurrent cardiac arrest: case report. Spinal Cord $35,256-257$

26. Eltorai, I., Kim, R., Vulpe, M., Kasravi, H., and Ho, W. (1992). Fatal cerebral hemorrhage due to autonomic dysreflexia in a tetraplegic patient: case report and review. Paraplegia 30, 355-360.

27. Vallès, M., Benito, J., Portell, E., and Vidal, J. (2005). Cerebral hemorrhage due to autonomic dysreflexia in a spinal cord injury patient. Spinal Cord 43, 738-740.

28. Jacob, C., Thwaini, A., Rao, A., Arya, N., Shergill, I.S., and Patel, H.R. (2005). Autonomic dysreflexia: the forgotten medical emergency. Hosp. Med. 66, 294-296.

29. Pan, S.-L., Wang, Y.-H., Lin, H.-L., Chang, C.-W., Wu, T.-Y., and Hsieh, E.-T. (2005). Intracerebral hemorrhage secondary to autonomic dysreflexia in a young person with incomplete C8 tetraplegia: a case report. Arch. Phys. Med. Rehabil. 86, 591-593.

30. Bycroft, J., Shergill, I.S., Choong, E.A., Arya, N., and Shah, J.R. (2005). Autonomic dysreflexia: a medical emergency. Postgr. Med. J. 81, 232-235.

31. Hainsworth, R., and Claydon, V.E. (2012). Syncope and fainting, in: Autonomic Failure, 5th ed. R. Bannister and C. Mathias (eds). Oxford University Press: Oxford, UK.

32. Furusawa, K., Tokuhiro, A., Sugiyama, H., Ikeda, A., Tajima, F., Genda, E., Uchida, R., Tominaga, T., Tanaka, H., Magara, A., and Sumida, M. (2011). Incidence of symptomatic autonomic dysreflexia varies according to the bowel and bladder management techniques in patients with spinal cord injury. Spinal Cord 49, 49-54.

33. Mathias, C.J. (2006). Orthostatic hypotension and paroxysmal hypertension in humans with high spinal cord injury. Prog. Brain Res. 152, 231-243.

34. Forrest, G.P. (1991). Atrial fibrillation associated with autonomic dysreflexia in patients with tetraplegia. Arch. Phys. Med. Rehabil. 72, 592-594.

35. Pine, Z.M., Miller, S.D., and Alonso, J.A. (1991). Atrial fibrillation associated with autonomic dysreflexia. Am. J. Phys. Med. Rehabil. 70, 271-273.

36. Kirshblum, S.C., House, J.G., and O'Connor, K.C. (2002). Silent autonomic dysreflexia during a routine bowel program in persons with traumatic spinal cord injury: a preliminary study. Arch. Phys. Med. Rehabil. 83, 1774-1776.

37. World Medical Association. (2008). Declaration of Helsinki-Ethical Principles for Medical Research Involving Human Subjects. World Medical Association: Ferney-Voltaire, France, pps., 1-8.

38. Krassioukov, A.V., Biering-Sorensen, F., Donovan, W., Kennelly, M., Kirshblum, S.C., Krogh, K., Alexander, M.S., Vogel, L., and Wecht, J. (2012). International standards to document remaining autonomic function after spinal cord injury. J. Spinal Cord Med. 35, 201-210.

39. Krogh, K., Perkash, I., Stiens, S., and Biering-Srensen, F. (2009). International bowel function extended spinal cord injury data set. Spinal Cord 47, 235-241.

40. Sahota, I.S., Ravensbergen, H.R., McGrath, M.S., and Claydon, V.E. (2012). Cerebrovascular responses to orthostatic stress after spinal cord injury. J. Neurotrauma 29, 2446-2456.

41. Juul, T., Bazzocchi, G., Coggrave, M., Johannesen, I., Hansen, R., Thiyagarajan, C., Poletti, E., Krogh, K., and Christensen, P. (2011). Reliability of the international spinal cord injury bowel function basic and extended data sets. Spinal Cord 49, 886-891.

42. Krupp, L.B., LaRocca, N.G., Muir-Nash, J., and Steinberg, A.D. (1989). The fatigue severity scale. Application to patients with multiple sclerosis and systemic lupus erythematosus. Arch. Neurol. 46, 1121-1123.

43. Griffin, K.A., Picken, M.M., and Bidani, A.K. (2004). Blood pressure lability and glomerulosclerosis after normotensive 5/6 renal mass reduction in the rat. Kidney Int. 65, 209-218. 
44. Kim, J., Koh, E., Leigh, J., and Shin, H.-I. (2012). Management of bowel dysfunction in the community after spinal cord injury: a postal survey in the Republic of Korea. Spinal Cord 50, 303-308.

45. Yim, S., Yoon, S., Lee, I., Rah, E., and Moon, H. (2001). A comparison of bowel care patterns in patients with spinal cord injury: upper motor neuron bowel vs lower motor neuron bowel. Spinal Cord 39, 204-207.

46. Cobb, J., Dumont, F., Leblond, J., Park, S.E., Noonan, V.K., and Noreau, L. (2014). An exploratory analysis of the potential association between SCI secondary health conditions and daily activities. Top. Spinal Cord Inj. Rehabil. 20, 277-288.

47. Sankari, A., Bascom, A., Oomman, S., and Badr, M.S. (2014). Sleep disordered breathing in chronic spinal cord injury. J. Clin. Sleep Med. $10,65-72$.

48. Lee, A.K., Miller, W.C., Townson, A.F., and Anton, H.A. (2010). Medication use is associated with fatigue in a sample of communityliving individuals who have a spinal cord injury: a chart review. Spinal Cord 48, 429-433.

49. Anton, H.A., Miller, W.C., and Townson, A.F. (2008). Measuring fatigue in persons with spinal cord injury. Arch. Phys. Med. Rehabil. $89,538-542$.

50. Fawkes-Kirby, T.M., Wheeler, M.A., Anton, H.A., Miller, W.C., Townson, A.F., and Weeks, C. (2008). Clinical correlates of fatigue in spinal cord injury. Spinal Cord 46, 21-25.

51. Jensen, M.P., Truitt, A.R., Schomer, K.G., Yorkston, K.M., Baylor, C., and Molton, I.R. (2013). Frequency and age effects of secondary health conditions in individuals with spinal cord injury: a scoping review. Spinal Cord 51, 882-892.

52. Alapin, I., Fichten, C.S., Libman, E., Creti, L., Bailes, S., and Wright, J. (2000). How is good and poor sleep in older adults and college students related to daytime sleepiness, fatigue, and ability to concentrate? J. Psychosom. Res. 49, 381-390.

53. Karlsson, A.K. (1999). Autonomic dysreflexia. Spinal Cord 37, 383-391.

54. Blackmer, J. (2003). Rehabilitation medicine: 1. Autonomic dysreflexia. Can. Med. Assoc. J. 169, 931-935.

55. Cragg, J., and Krassioukov, A. (2012). Autonomic dysreflexia. Can. Med. Assoc. J. 184, 66.

56. Wecht, J.M., Weir, J.P., DeMeersman, R.E., Spungen, A.M., and Bauman, W.A. (2004). Arterial stiffness in persons with paraplegia. J. Spinal Cord Med. 27, 255-259.

57. Elliott, S., and Krassioukov, A. (2006). Malignant autonomic dysreflexia in spinal cord injured men. Spinal Cord 44, 386-392.

58. Goh, M.Y., Wong, E.C., Millard, M.S., Brown, D.J., and O'Callaghan, C.J. (2015). A retrospective review of the ambulatory blood pressure patterns and diurnal urine production in subgroups of spinal cord injured patients. Spinal Cord 53, 49-53.

59. Consortium for Spinal Cord Medicine. (2002). Acute management of autonomic dysreflexia: individuals with spinal cord injury presenting to health-care facilities. Paralyzed Veterans of America: Washington, DC.
60. CareCure Community. (2010). Wife learning bowel program for husband.Available at: http://sci.rutgers.edu/forum/showthread.php?t= 1328132010 (last accessed July 19, 2017).

61. Furusawa, K., Sugiyama, H., Tokuhiro, A., Takahashi, M., Nakamura, T., and Tajima, F. (2009). Topical anesthesia blunts the pressor response induced by bowel manipulation in subjects with cervical spinal cord injury. Spinal Cord 47, 144-148.

62. Wecht, J.M., and Bauman, W.A. (2013). Decentralized cardiovascular autonomic control and cognitive deficits in persons with spinal cord injury. J. Spinal Cord Med. 36, 74-81.

63. Jegede, A.B., Rosado-Rivera, D., Bauman, W.A., Cardozo, C.P., Sano, M., Moyer, J.M., and Wecht, J.M. (2010). Cognitive performance in hypotensive persons with spinal cord injury. Clin. Auton. Res 20, 3-9.

64. Wecht, J.M., Rosado-Rivera, D., Jegede, A.B., Cirnigliaro, C.M., Jensen, M.A., Kirshblum, S., and Bauman, W.A. (2012). Systemic and cerebral hemodynamics during cognitive testing. Clin. Auton. Res 22, 25-33.

65. Ghaisas, S., Pyatak, E.A., Blanche, E., Blanchard, J., Clark, F., and Chan, T.H. (2015). Lifestyle changes and pressure ulcer prevention in adults with spinal cord injury in the pressure ulcer prevention study lifestyle intervention. Am. J. Occup. Ther. 69, 1-10.

66. Westerkam, D., Saunders, L.L., and Krause, J.S. (2011). Association of spasticity and life satisfaction after spinal cord injury. Spinal Cord 49, 990-994.

67. Consortium for Spinal Cord Medicine. (1999). Neurogenic bowel: What you should know. Paralyzed Veterans of America: Washington, DC.

68. Shepherd, J.D., Badger-Brown, K.M., Legassic, M.S., Walia, S., and Wolfe, D.L. (2012). SCI-U: E-learning for patient education in spinal cord injury rehabilitation. J. Spinal Cord Med. 35, 319-329.

69. Kowalczewski, J., Ling Chong, S., Galea, M., and Prochazka, A (2011). In-home tele-rehabilitation improves tetraplegic hand function. Neurorehabil. Neural Repair 25, 412-422.

70. Houlihan, B.V, Jette, A., Friedman, R.H., Paasche-Orlow, M., Ni, P., Wierbicky, J., Williams, K., Ducharme, S., Zazula, J., Cuevas, P., Rosenblum, D., and Williams, S. (2013). A pilot study of a telehealth intervention for persons with spinal cord dysfunction. Spinal Cord 51, $715-720$.

Address correspondence to:

Victoria E. Claydon, PhD

Department of Biomedical Physiology and Kinesiology

Faculty of Science

Simon Fraser University 8888 University Drive

Burnaby, British Columbia V5A 1 S6

Canada

E-mail: victoria_claydon@sfu.ca 